\title{
HIGH-PERFORMANCE RADIATION DESIGN OF A PLANAR BOW-TIE ANTENNA COMBINED WITH A DIELECTRIC LENS AND CASCADED MATCHING LAYERS AT TERAHERTZ FREQUENCIES
}

\author{
Catur Apriono $^{1 *}$, Arie Pangesti Aji ${ }^{1}$, Teguh Wahyudi ${ }^{1}$, Fitri Yuli Zulkifli ${ }^{1}$, Eko Tjipto Rahardjo ${ }^{1}$ \\ ${ }^{1}$ Antenna Propagation and Microwave Research Group (AMRG), Department of Electrical Engineering, \\ Faculty of Engineering, Universitas Indonesia, Kampus UI Depok, Depok 16424, Indonesia
}

(Received: November 2017 / Revised: February 2018 / Accepted: April 2018)

\begin{abstract}
The demand for high-speed data transmission has increased significantly in the last decades. Terahertz (THz) frequencies, which lie between $100 \mathrm{GHz}$ to $10 \mathrm{THz}$, have been considered to be the solution to the demand. However, the low gain and low efficiency of a $\mathrm{THz}$ antenna remain issues that hinder reasonable performance for various applications. This paper proposes the design of a high-gain and high-efficiency planar bow-tie antenna for applications using $\mathrm{THz}$ frequencies. A planar bow-tie on a high-resistivity silicon substrate is considered in order to obtain broadband characteristics. To increase gain and efficiency, a dielectric silicon lens and a matching layer based on the quarter-wavelength are applied in the design. From simulations using Computer Simulation Technology (CST) Microwave Studio, gain and radiation efficiency of up to $32.69 \mathrm{~dB}$ and $90.4 \%$ are obtained, respectively. This proposed design has shown high radiation performance suitable for high-speed transmission systems.
\end{abstract}

Keywords: Bow-tie; High resistivity silicon lens; Matching layer; Terahertz

\section{INTRODUCTION}

The terahertz $(\mathrm{THz})$ wave radiation region, located between the microwave and the infrared regions, is the transition region between the electronic and photonic domains (Akyildiz et al., 2014). The THz wave is not applied as widely as the electronic and photonic domains, although there is potential for this frequency band. The availability of reasonable performance equipment, especially for $\mathrm{THz}$ wave sources and detectors, is one of the main issues of $\mathrm{THz}$ wave system development. As femtosecond lasers and photoconductive antennas have been available since the 1980s to produce THz waves (Mourou et al., 1981), some areas have begun to utilize these waves, such as medical science, pharmacology, and security (Siegel, 2002). This region has also been considered to fulfil the demand for high-speed wireless data transmission and various communication applications (Hirata et al., 2012). High-speed data transmission demand has increased significantly in the last decades. Some modifications have been made to improve data capacity by applying advanced modulation schemes and signal processing. In the future, a new spectral resource is needed to increase data speed. The large bandwidth of $\mathrm{THz}$ waves can be utilized to obtain higher data rates, even with the legacy data modulation technique. Therefore, a THz wave carrier frequency is necessary to increase bandwidth and fulfil requirements (Song \& Nagatsuma, 2011).

Despite its advantages of high speed and large bandwidth, THz wave radiation has a significant

\footnotetext{
*Corresponding author's email catur@eng.ui.ac.id, Tel. +62-21-7270078, Fax.+62-21-7270077

Permalink/DOI: https://dx.doi.org/10.14716/ijtech.v9i3.955
} 
amount of signal loss compared to the microwave frequency band. The atmospheric attenuation at $\mathrm{THz}$ frequencies is too large for long range outdoor wireless communications (ITU-R Recommendation, 2009). However, this system still can be used as an alternative solution for future short-range indoor wireless communication, such as WLANs or WPANs, to provide data rates of ten Gbps and beyond (Song \& Nagatsuma, 2011). Theoretically, the issue can be minimized by providing a sufficient transmitted source power or a high-gain antenna, especially for outdoor application. The concept of a $\mathrm{THz}$ antenna is expected to be an effective method to produce $\mathrm{THz}$ wave radiation, which has already been applied and is mature in the electronic domain.

Among the various types of antennas, a bow-tie type is often considered as a broad bandwidth antenna. The bi-conical structure of this type of antenna is easy to design and is affordable (Kaur \& Solanki, 2012). It consists of two triangles facing each other from their apex, with a specific gap to form a dipole antenna. The performance of the bow-tie antenna depends on its geometrical parameters, including bow-tie length, apex angle and gap size. In addition, a flexible bow-tie antenna is expected to be more directional than a conventional dipole antenna because of its larger radiating area (Durgun et al., 2011).

In a THz system, an antenna can be used by combining optical elements, such as a lens or mirror, to provide reasonable radiation performance for quasi-optical detectors or radiators (Hesler et al., 2008). The bow-tie antenna combined with a capacitive bar and a hemispherical silicon lens has been studied for broadband applications; however, the radiation characteristics of gain and efficiency should be improved to achieve more reasonable radiation performance (Wahyudi et al., 2017). A bow-tie photoconductive antenna on GaAs substrate has been studied for the $\mathrm{THz}$ frequency band, and its directivity performance can be improved by using a silicon lens (Jyothi et al., 2016). Surface wave suppression has been studied by using bow-tie THz antennas on InP substrate combined with hemisphere and bullet Si lens, which has led to higher gain and efficiency and also broader bandwidth (Li \& Song, 2016). A dipole bow-tie slot antenna on $\mathrm{Si}$ substrate for imaging application at $94 \mathrm{GHz}$ has been studied by using $\mathrm{SiO}_{2}$ as an insulating dielectric material to enhance gain and side lobe level performances (Haraz et al., 2014).

This paper proposes a high-gain and high-efficiency bow-tie planar antenna design for applications in the $\mathrm{THz}$ frequencies. A planar bow-tie antenna on a high resistivity silicon substrate is considered in order to obtain broadband characteristics. The bow-tie antenna is designed to achieve a $1 \mathrm{THz}$ resonant frequency. Its design is then modified by applying a capacitive bar attached near its apex points to widen the bandwidth (Zhang et al., 2016). To increase the gain and radiation efficiency, a dielectric silicon lens and a matching layer based on the quarter-wavelength are applied in the design. The substrate thickness is also parameterized to obtain the highest gain. The proposed antenna is expected to achieve high gain and efficiency application in the $\mathrm{THz}$ region for both indoor and outdoor applications. Therefore, we expect to obtain a high-performance $\mathrm{THz}$ antenna design which can provide gain, bandwidth and efficiency of at least $30 \mathrm{~dB}, 20 \%$ and $90 \%$, respectively. The desired gain is intended to provide a communication link range at least $10 \mathrm{~m}$ in a $\mathrm{THz}$ communication system which is largely attenuated by atmospheric loss (Song \& Nagatsuma, 2011), and the expected bandwidth to follow the ultra-wideband (UWB) system, which requires bandwidth at least $20 \%$ from its resonant frequency center. The desired efficiency is based on the performance of the antireflection coating technique, which can reduce internal reflection in the lens antenna and thus increase the radiation efficiency. Design and simulation are performed with Computer Simulation Technology (CST) Microwave Studio software. This simulator is one of the most well-known electromagnetic (EM) softwares and is widely used by many researchers in the field of electromagnetics. The $\mathrm{THz}$ antenna needs to be fabricated in the micro- or nanometer 
scale due to its wavelength. Due to fabrication difficulties and limited THz measurement tools, we consider simulation results and theoretical study to analyze the research. Designs using this software have been made by many researchers and validated by measurement in the microwave research area. Therefore, we can rely on the simulation results to analyze our proposed design.

\section{ANTENNA DESIGN}

\subsection{Planar Bow-tie Antenna}

Figure 1 illustrates a schematic of the bow-tie antenna. It consists of two identical triangles separated by a specific gap distance. A discrete port with a $50 \Omega$ input impedance is used as the feeding method, set in the gap. High-resistivity silicon with a relative dielectric constant $\left(\varepsilon_{r}\right)$ set to 11.9 was chosen due to its low refractive index dispersion and low absorption, which are suitable for electromagnetic wave radiations (Ronne et al., 1997). The thickness of the silicon wafer substrate of $300 \mu \mathrm{m}$ is considered to comply with the availability of Si wafers on the market. The geometrical parameters of the antenna include antenna length $(l)$, width $(w)$, gap $(g)$, flare angle $(\theta)$, bar length $(b l)$, and bar width $(b w)$. The substrate dimensions consist of length $(s)$ and thickness $(h)$. As the bow-tie antenna performance can be treated as that of a dipole type antenna, $1 \mathrm{THz}$ resonant frequency can be obtained by simply using half wavelength-dipole calculation or its periodic length pattern, such as $\lambda / 2,3 / 2 \lambda, 5 / 2 \lambda$, and so on (Wang \& Zhan, 2013). The parameters of the antenna and the substrate are characterized to obtain a resonant frequency of $1 \mathrm{THz}$. Table 1 shows the optimum parameters after characterization for a resonant frequency of $1 \mathrm{THz}$. The length of the bow-tie antenna $(l)$ is close to $3 / 2 \lambda$ of a $1 \mathrm{THz}$ frequency. At a resonant frequency of $1 \mathrm{THz}$, the $300 \mu \mathrm{m}$ wavelength becomes shorter $\left(\lambda=\lambda_{0} / \sqrt{\varepsilon_{r}}\right)$. When the waves propagate in the silicon material (11.9), the wavelength is about $87 \mu \mathrm{m}$. As the planar antenna radiates most of its energy to the substrate side, it can lead to a surface wave problem on this side (Rebeiz, 1992). Therefore, the substrate dimension and thickness values are considered in order to deal with the substrate wave.

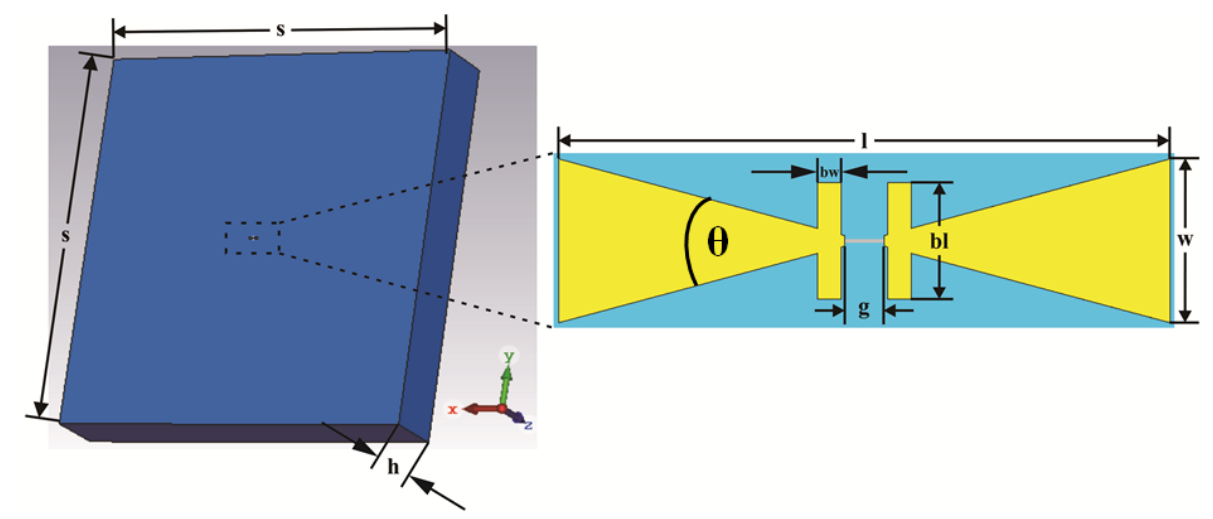

Figure 1 Schematic of the bow-tie antenna design on a high resistivity silicon substrate

Table 1 Antenna and substrate parameters

\begin{tabular}{cr}
\hline Parameter & \multicolumn{1}{c}{ Value } \\
\hline$S$ & $6000 \mu \mathrm{m}$ \\
$H$ & $300 \mu \mathrm{m}$ \\
$L$ & $156.8 \mu \mathrm{m}$ \\
$W$ & $42 \mu \mathrm{m}$ \\
$b w$ & $6 \mu \mathrm{m}$ \\
$b l$ & $30 \mu \mathrm{m}$ \\
$G$ & $10 \mu \mathrm{m}$ \\
$\theta$ & $30^{\circ}$ \\
\hline
\end{tabular}




\subsection{Silicon Dielectric Lens}

An antenna placed on a thick dielectric substrate will radiate most of its energy into the dielectric side (Rebeiz, 1992). A hemispherical shape is used to minimize the internal reflection as well as the surface waves, so that radiation efficiency is increased. Figure 2 illustrates a dielectric lens designed on top of the substrate. To form a hemisphere shape, the lens radius $(R)$ is set to $3000 \mu \mathrm{m}$, or half the dimension of the Si substrate $(s)$. The center point of the lens is at the top of the substrate, or at the point of substrate thickness $(h)$. The lens dielectric constant is determined to be same as the substrate dielectric constant to avoid a mismatched condition between the two.

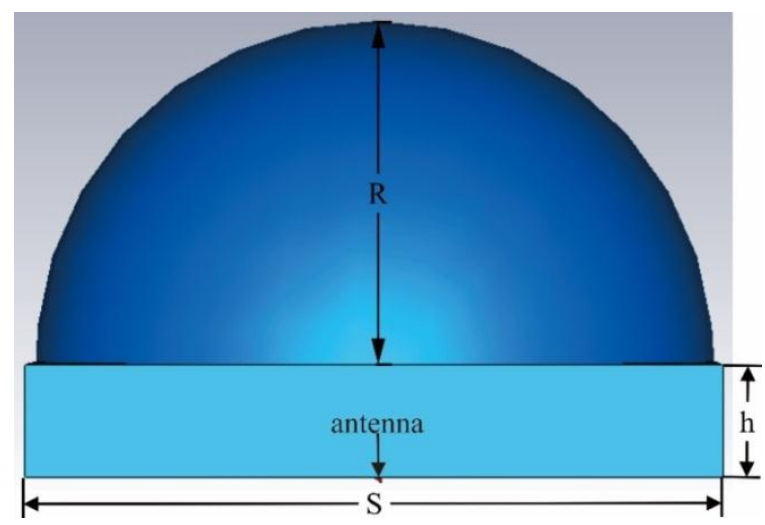

Figure 2 Schematic of a dielectric lens structure

\section{RESULTS AND DISCUSSION}

\subsection{Planar Bow-tie Antenna}

This section discusses the simulation results of the planar bow-tie antenna shown in Figure 2. The discussion is conducted for two parameters, i.e. return loss and radiation pattern.

\subsubsection{Return loss (S11) parameter}

Figure 3 shows the S11 simulation results of the bow-tie antenna. The line is not smooth, which indicates internal reflections occurring in the substrate. This issue can be solved by adding a hemispherical lens to provide a radiation vector parallel to the hemispherical surface vector. The return loss at $1 \mathrm{THz}$ is around $-20 \mathrm{~dB}$. The bandwidth, which is determined at the threshold of $-10 \mathrm{~dB}$, is $364.97 \mathrm{GHz}$. Therefore, the result shows that the antenna's resonant frequency is $1 \mathrm{THz}$ and has broad bandwidth characteristics, as expected from a conventional bow-tie antenna design.

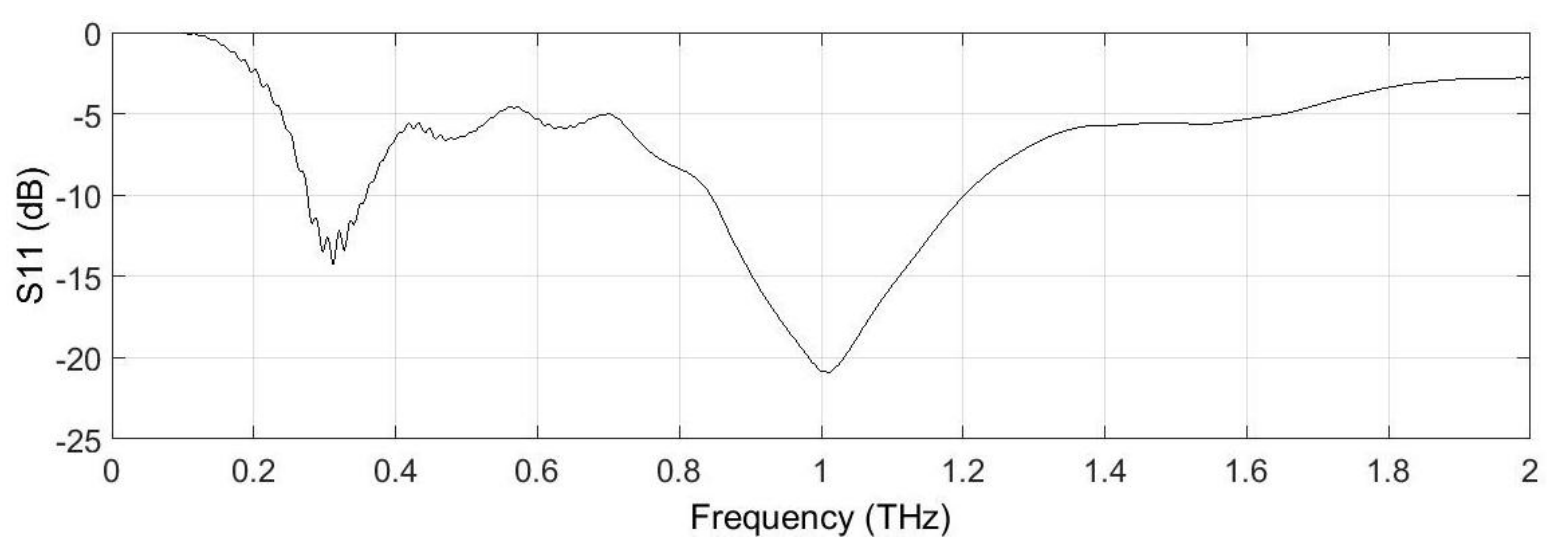

Figure 3 S11 parameter simulation results of the initial bow-tie antenna design 


\subsubsection{Radiation pattern}

Figure 4 shows the radiation pattern simulation results at $1 \mathrm{THz}$ frequency for planes of (a) $\phi=$ $0^{\circ}$ (E-plane) and (b) $\phi=90^{\circ}$ (H-plane), respectively. The red and green lines represent the gain levels for all directions and the side lobe level, respectively. Both figures show that the radiation patterns are not as desired. This issue is related to the internal reflections, which lead to the radiated power being trapped in the substrate or surface waves. The problem can be solved by considering infinite substrate thickness or by providing a substrate surface with normal vectors to all radiation vectors. We consider the latter solution to solve this issue. The realized gain is $-4.631 \mathrm{~dB}$. The radiation efficiency and total efficiency are $-13.97 \mathrm{~dB}(4 \%)$ and $-14.01 \mathrm{~dB}(3.9 \%)$, respectively. The radiation pattern seems to be omnidirectional. The main lobe is directed at $-53^{\circ}$ (E-plane) and $56^{\circ}$ (H-plane), which means that the antenna cannot radiate the energy directly to the desired direction $\left(0^{\circ}\right)$ from its center point. Although the angular beam width is narrow at the main lobe, these results indicate that the antenna is not applicable for implementation in practical use, because most of the power radiated from it is reflected back into the substrate, while the radiated power to free space is very low.

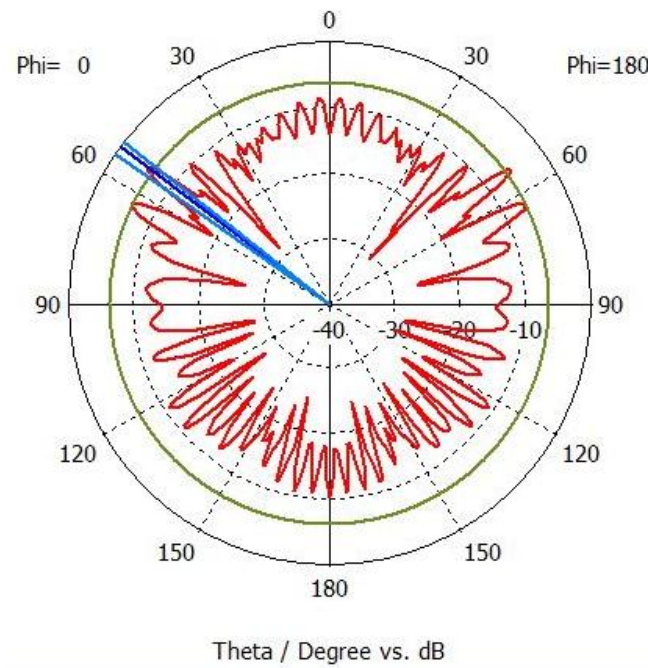

(a)

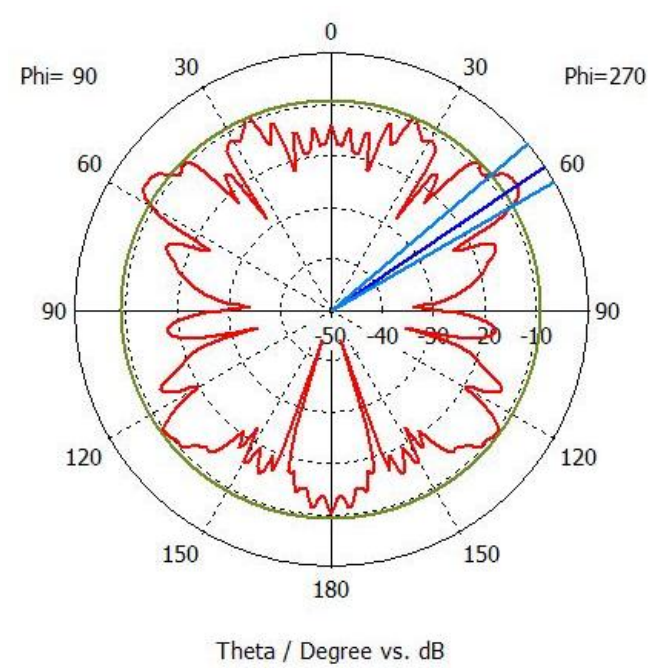

(b)

Figure 4 Radiation patterns of the bow-tie antenna for: (a) $\phi=0^{\circ}$; and (b) $\phi=90^{\circ}$

\subsection{Bow-tie Antenna Combined with a Dielectric Lens}

This section discusses the simulation results of the bow-tie antenna combined with a dielectric lens, as shown in Figure 2. The discussion relates to two parameters, return loss and radiation pattern.

\subsubsection{Return loss (S11) parameter}

Figure 5 shows the S11 simulation results of the bow-tie antenna combined with a silicon dielectric lens. The resonant frequency of $1 \mathrm{THz}$ remains at around $-20 \mathrm{~dB}$, while the bandwidth determined at the threshold of $-10 \mathrm{~dB}$ is $368.76 \mathrm{GHz}$. The $\mathrm{S} 11$ parameter shows that the antenna is still able to resonate at $1 \mathrm{THz}$ and has broadband characteristics. These results indicate that the addition of the dielectric lens changes neither resonance nor bandwidth. The return loss line in Figure 5 is smoother than that in Figure 4, implying that the surface waves have been successfully minimized by the combination of the bow-tie antenna with the dielectric lens. The plot of gain for some frequencies shows that the gain fluctuates around $10 \mathrm{~dB}$, and the highest gain is not at the resonant frequency of $1 \mathrm{THz}$. We expect the highest gain to be at the resonant frequency. Therefore, this structure is not yet at the optimum condition. In subsection 
3.3 we investigate the optimum gain by characterizing substrate thickness. This technique is intended to find a focal position to produce higher directivity.

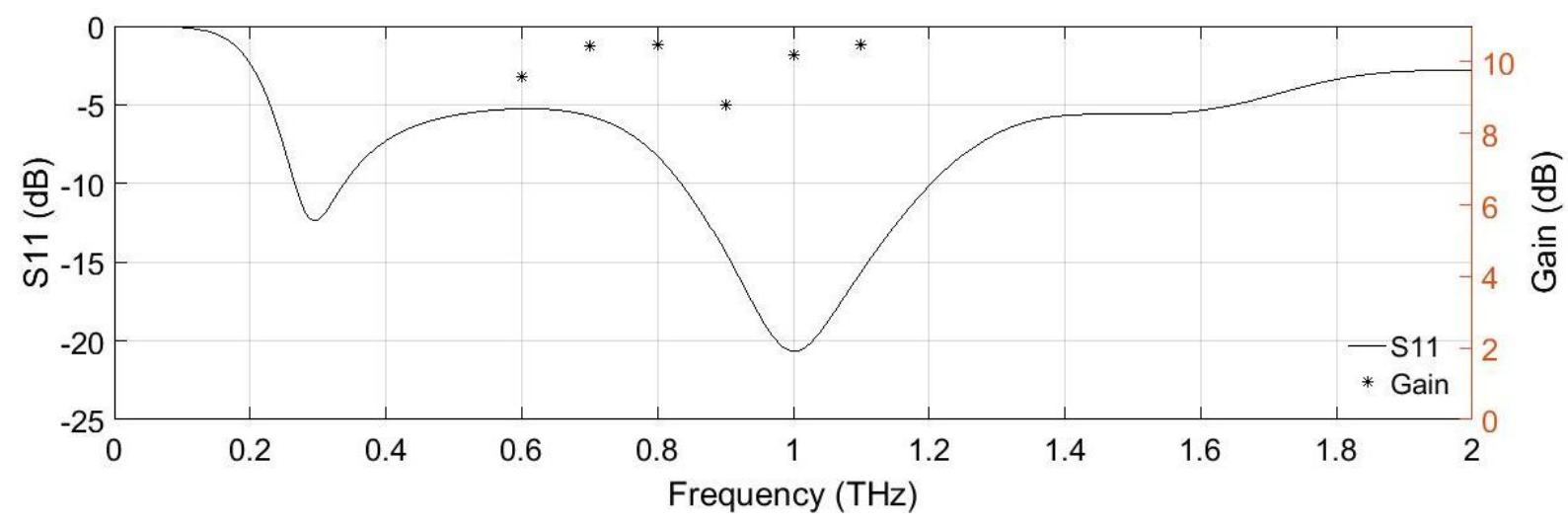

Figure 5 S11 Parameter simulations of the bow-tie antenna combined with dielectric lens

\subsubsection{Radiation pattern}

Figure 6 shows the radiation pattern simulation results of the bow-tie antenna combined with a dielectric lens at $1 \mathrm{THz}$ for a plane of (a) $\phi=0^{\circ}$ (E-plane) and (b) $\phi=90^{\circ}$ (H-plane). The highest realized gain is $10.18 \mathrm{~dB}$, obtained from the main lobe magnitude of Figure $6 \mathrm{a}$. The radiation and total efficiency are $-1.592 \mathrm{~dB}(69.3 \%)$ and $-1.63 \mathrm{~dB}(68.7 \%)$, respectively. The realized gain and efficiencies were obtained from the $3 \mathrm{D}$ farfield simulation results at the frequency of $1 \mathrm{THz}$. The results indicate that the dielectric lens has increased the efficiency and gain performance of the antenna; nevertheless, the main lobe direction and angular width are not suitable for the antenna to radiate its power with high directivity in a desired direction. Therefore, an optimization process is necessary to improve the results. In the following steps, substrate thickness characterization and multilayer antireflection coating were applied to improve the results.

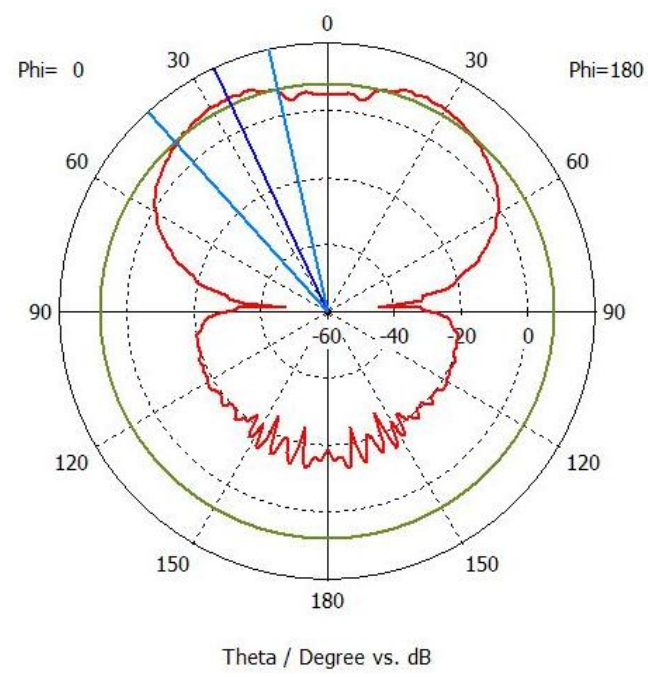

(a)

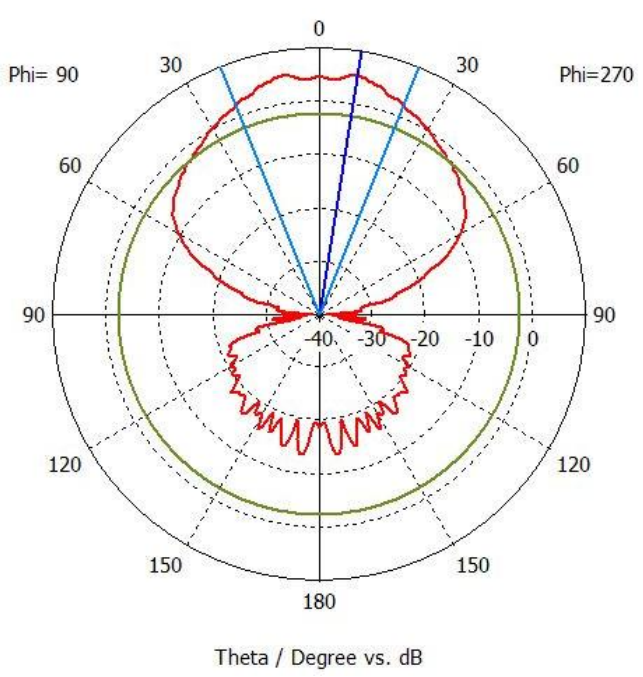

(b)

Figure 6 Radiation pattern of the bow-tie antenna combined with dielectric lens at: (a) $\phi=0^{\circ}$ (E-plane); and (b) $\phi=90^{\circ}$ (H-plane)

\subsection{Substrate Thickness Characterization}

In this stage, optimization is conducted to further improve the directivity by varying the substrate thickness. The antenna and dielectric lens geometry are kept constant, as detailed in 
Table 1, and the iteration in thickness is conducted from $300 \mu \mathrm{m}$ to $1100 \mu \mathrm{m}$. The effect of thickness will be discussed in terms of gain, efficiency and angular beam width.

\subsubsection{Gain}

Figure 7 shows the gain for different substrate thicknesses. The gain increases for thicknesses of $300 \mu \mathrm{m}$ to $1000 \mu \mathrm{m}$ and subsequently decreases. The maximum gain of $31.31 \mathrm{~dB}$ is obtained at a thickness of $1000 \mu \mathrm{m}$ and produces a significant gain improvement until $20 \mathrm{~dB}$ compared to the initial thickness. High gain is necessary to mitigate the high attenuation when the radiation propagates in the atmospheric free space. The results show that the substrate thickness affects the antenna's directivity, and the optimum gain can be obtained at a certain thickness.

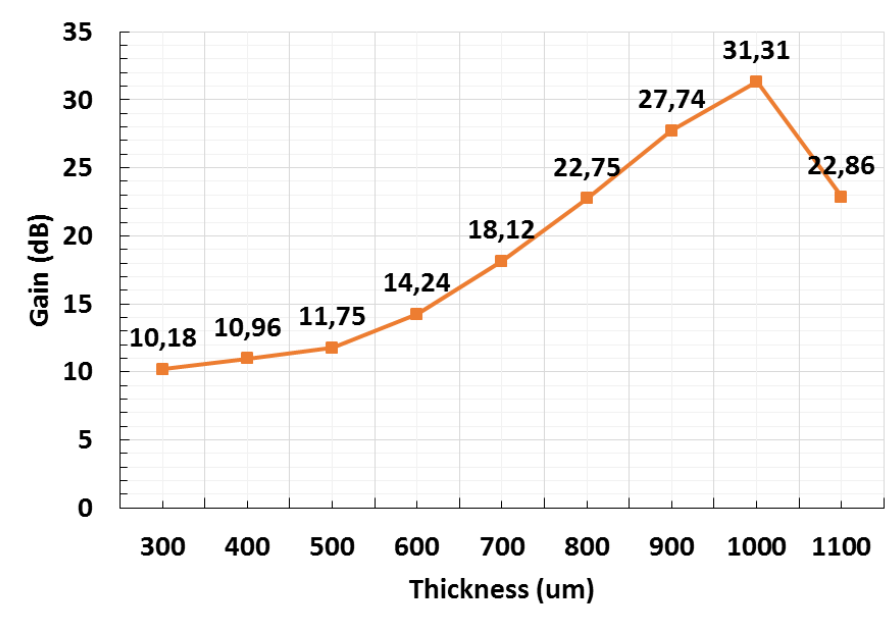

Figure 7 Gain versus substrate thickness

\subsubsection{Radiation efficiency}

Figure 8 shows the radiation efficiency for different substrate thicknesses; a maximum efficiency of $71.8 \%$ is obtained at the substrate thickness of $900 \mu \mathrm{m}$, but efficiency starts to drop from a thickness of $1000 \mu \mathrm{m}$ and beyond. From the results, we can conclude that substrate thickness has a slight effect on the antenna's efficiency. This condition comes from the radiation wavefront conforming with the lens surface, where the optimum thickness to pass through the interface is around $900 \mu \mathrm{m}$.

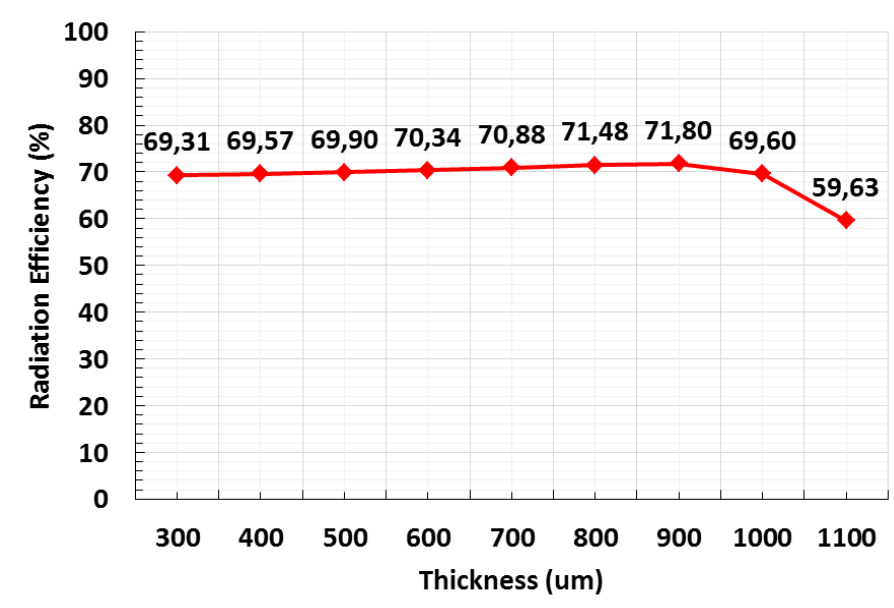

Figure 8 Radiation efficiency versus substrate thickness

\subsubsection{Angular beamwidth}

Figure 9 shows the beam width at plane $\phi=90^{\circ}$ versus the substrate thickness. The smallest beam width is $3.1^{\circ}$, obtained at a thickness of $1000 \mu \mathrm{m}$. The results indicate that the substrate 
thickness causes the radiation pattern to be more directive when the beam width becomes narrower. The narrow beam width pattern is useful in providing high-resolution imaging when the antenna is used in $\mathrm{THz}$ imaging applications.

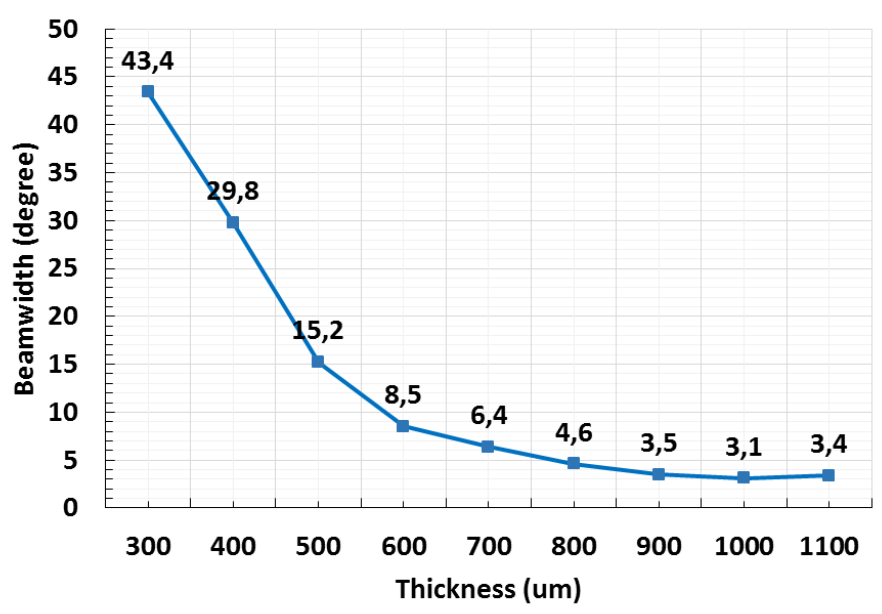

Figure 9 Beamwidth versus substrate thickness

\subsection{Antireflection coating implementation}

A common problem with lens antennas is that the incident wave is partially reflected back to the substrate at the lens-air interface, thereby affecting radiation efficiency (van der Vorst et al., 1999). Studies on canceling internal reflection in a dielectric lens have been performed since 1954 using quarter-wave antireflection coating (Morita \& Cohn, 1956). The coating works according to the principle of phase changing between the reflected waves and depends on the different refractive indices of the lens and the coating. The phase changing results from ray propagation through the coating, and because of the different path lengths between subsequent rays which leave the substrate at different points, based on a far-field observation point. The coating is theoretically designed using an infinite number of internal reflections. The derivation of the infinite reflection and transmission coefficients use the model and configuration described by van Houten \& Herben (1994). In this section, an antireflection coating, otherwise known as a matching layer (ML), is designed using different techniques.

\subsubsection{Quarter-wave matching layer}

Figure 10 shows the proposed ML structure. The dielectric constant $\left(\varepsilon_{e}\right)$, effective wavelength $\left(\lambda_{e}\right)$ and thickness $(d)$ of the quarter-wave ML are obtained by Equations 1,2 and 3, respectively. $\varepsilon_{0}$ is the dielectric constant of air, which is one. $\lambda_{0}$ is the wavelength in free space for the $1 \mathrm{THz}$ frequency, and $\lambda_{e}$ is the effective wavelength of the ML. By using 11.9 as the silicon dielectric constant $\left(\varepsilon_{s}\right)$, the effective dielectric constant $\left(\varepsilon_{e}\right)$ and the layer thickness $(d)$ are 3.45 and $40.4 \mu \mathrm{m}$, respectively, as calculated by Equations 1 to 3 (van der Vorst et al., 1999). The geometry of the antenna and dielectric lens are kept constant, as mentioned in section 2. The substrate thickness $(h)$ is $1000 \mu \mathrm{m}$ and is obtained from the thickness of the optimum gain and beam width in section 3 .

$$
\begin{aligned}
\varepsilon_{e} & =\sqrt{\varepsilon_{s} \cdot \varepsilon_{0}} \\
\lambda_{e} & =\lambda_{0} / \varepsilon_{e} \\
d & =\lambda_{e} / 4
\end{aligned}
$$




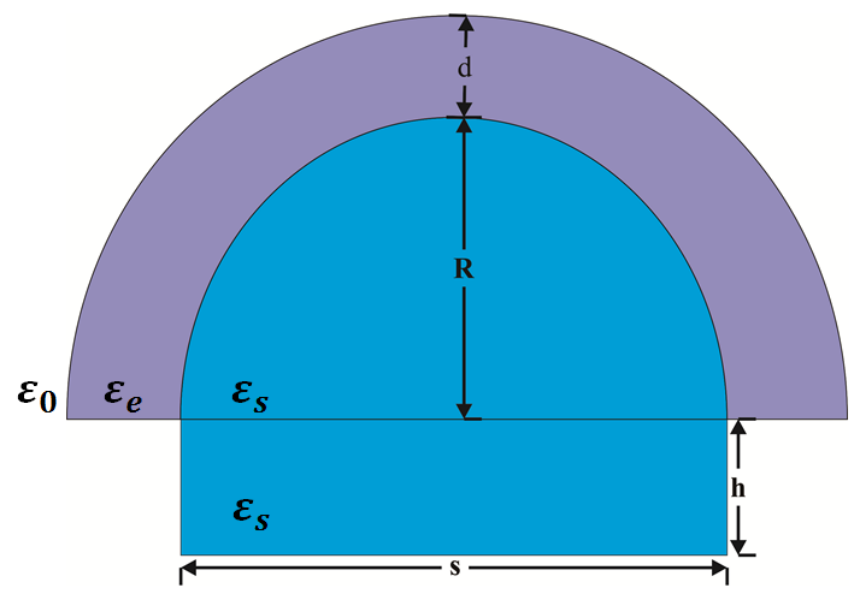

Figure 10 Schematic of the matching layer design

From the design shown in Figure 10, the radiation efficiency and gain have increased to $83.1 \%$ and $32.31 \mathrm{~dB}$ respectively. It is clear that the matching layer has lowered the internal reflection significantly, but not completely. The efficiency improvement is not as good as that reported by van der Vorst et al. (1999), because the antenna already has a narrow beam width and good directivity. In addition, the proposed lens antenna has different angles of incident at every different surface position. Therefore, the single quarter-wavelength matching layer does not completely reduce the reflection effect on the lens antenna, because it can only reduce the internal reflection if the wave vector is parallel to the normal vector. An optimum matching layer with non-uniform thickness is more preferable, but we do not study this because it is difficult to design and not practical in real conditions. We consider a cascaded matching layer, which will be discussed in the following section.

\subsubsection{Cascaded matching layer}

The concept of the cascaded matching layer is the same as that of the quarter-wave in the previous subsection, but with multiple-layer implementation. The dielectric constant and thickness of each layer can still be obtained using Equations 1 to 3, derived into Equation 4.

$$
\varepsilon_{m}=\varepsilon_{s}^{(n+1)-m /(n+1)}
$$

$\varepsilon_{m}$ is the dielectric constant of every layer in an $n$-ML design and $\varepsilon_{s}$ is the dielectric constant of the substrate material. The effective wavelength and thickness of every layer can be obtained using the dielectric constant obtained from Equation 4. Theoretically, a higher number of matching layers corresponds to better performance in terms of radiation characteristics such as gain and efficiency (Tokan, 2014). In this paper, the effects of 15 matching layers are studied, to find an ideal number based on their performance. A sample schematic of a ten-layer design of the cascaded matching layer is shown in Figure 11, which depicts the layers stacked on top of the dielectric lens. To avoid overlapping layers, an insertion technique is applied in CST. The thickness of each layer obtained from Equation 4 is distinct, where the higher-level layer is thicker. Other dimensions of the design are kept constant, as in the previous design. The complete list of dielectric constants of the cascaded MLs from one ML to 15 MLs is summarized in Table 2.

As shown in Figure 12, the radiation efficiency shows incremental values from one ML to 15 MLs, with a maximum value of $93.7 \%$ at 15 layers. The results show that a higher number of MLs can reduce more internal reflection, thus increasing the radiation efficiency. The gain value also shows incremental values from one ML to four MLs, with a maximum value of $32.81 \mathrm{~dB}$, but starts to decrease significantly as the MLs become larger. This shows that four 
MLs is the upper limit for achieving the highest gain. This may be affected by refraction at every boundary between two dielectrics. More MLs increases the level of refraction. Eventually, the higher number of cascaded MLs corresponds to a tradeoff between radiation efficiency and gain, which shows an intersection between radiation efficiency and gain at five layers as the optimum number of cascaded matching layers, with values of $90.4 \%$ and 32.69 $\mathrm{dB}$, respectively.

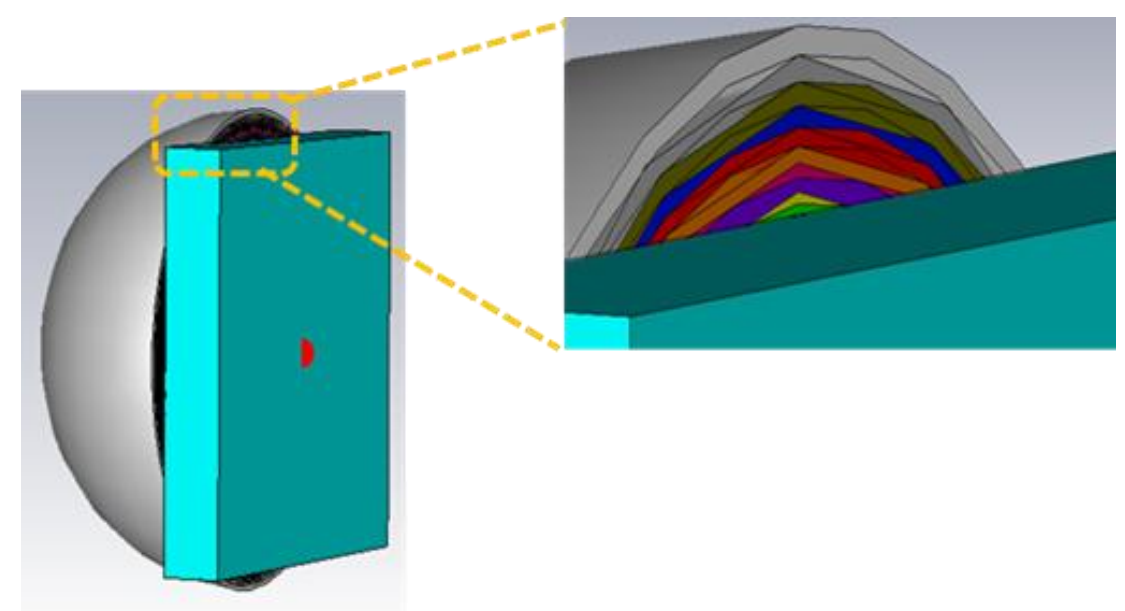

Figure 11 Cascaded matching layer design

Table 2 Dielectric constant of cascaded matching layer

\begin{tabular}{lllllllllllllllllll}
\hline $\mathbf{n - M L} \backslash \boldsymbol{\varepsilon}_{\mathbf{m}}$ & $\varepsilon 1$ & $\varepsilon 2$ & $\varepsilon 3$ & $\varepsilon 4$ & $\varepsilon 5$ & $\varepsilon 6$ & $\varepsilon 7$ & $\varepsilon 8$ & $\varepsilon 9$ & $\varepsilon 10$ & $\varepsilon 11$ & $\varepsilon 12$ & $\varepsilon 13$ & $\varepsilon 14$ & $\varepsilon 15$ \\
\hline $1 \mathrm{ML}$ & 3.45 & - & - & - & - & - & - & - & - & - & - & - & - & - & - \\
$2 \mathrm{ML}$ & 5.21 & 2.28 & - & - & - & - & - & - & - & - & - & - & - & - & - \\
$3 \mathrm{ML}$ & 6.41 & 3.45 & 1.86 & - & - & - & - & - & - & - & - & - & - & - & - \\
$4 \mathrm{ML}$ & 7.25 & 4.42 & 2.69 & 1.64 & - & - & - & - & - & - & - & - & - & - & - \\
$5 \mathrm{ML}$ & 7.88 & 5.21 & 3.45 & 2.28 & 1.51 & - & - & - & - & - & - & - & - & - & - \\
$6 \mathrm{ML}$ & 8.35 & 5.86 & 4.12 & 2.89 & 2.03 & 1.42 & - & - & - & - & - & - & - & - & - \\
$7 \mathrm{ML}$ & 8.73 & 6.41 & 4.70 & 3.45 & 2.53 & 1.86 & 1.36 & - & - & - & - & - & - & - & - \\
$8 \mathrm{ML}$ & 9.04 & 6.86 & 5.21 & 3.96 & 3.01 & 2.28 & 1.73 & 1.32 & - & - & - & - & - & - & - \\
$9 \mathrm{ML}$ & 9.29 & 7.25 & 5.66 & 4.42 & 3.45 & 2.69 & 2.10 & 1.64 & 1.28 & - & - & - & - & - & - \\
$10 \mathrm{ML}$ & 9.50 & 7.59 & 6.06 & 4.84 & 3.86 & 3.08 & 2.46 & 1.96 & 1.57 & 1.25 & - & - & - & - & - \\
$11 \mathrm{ML}$ & 9.68 & 7.88 & 6.41 & 5.21 & 4.24 & 3.45 & 2.81 & 2.28 & 1.86 & 1.51 & 1.23 & - & - & - & - \\
$12 \mathrm{ML}$ & 9.84 & 8.13 & 6.72 & 5.55 & 4.59 & 3.79 & 3.14 & 2.59 & 2.14 & 1.77 & 1.46 & 1.21 & - & - & - \\
$13 \mathrm{ML}$ & 9.97 & 8.35 & 7.00 & 5.86 & 4.91 & 4.12 & 3.45 & 2.89 & 2.42 & 2.03 & 1.70 & 1.42 & 1.19 & - & - \\
$14 \mathrm{ML}$ & 10.09 & 8.55 & 7.25 & 6.15 & 5.21 & 4.42 & 3.75 & 3.18 & 2.69 & 2.28 & 1.94 & 1.64 & 1.39 & 1.18 & - \\
$15 \mathrm{ML}$ & 10.19 & 8.73 & 7.48 & 6.41 & 5.49 & 4.70 & 4.03 & 3.45 & 2.95 & 2.53 & 2.17 & 1.86 & 1.59 & 1.36 & 1.17 \\
\hline
\end{tabular}

\subsection{Theoretical Validation}

This subsection discusses the theoretical calculation to validate the results of the proposed design. The standard for gain can be defined by Equation 5. Gain is a function of elevation $(\theta)$ and azimuth angle $(\phi) . U(\theta, \phi)$ is the radiation intensity and $P_{i n}$ is the input power to the antenna.

$$
G(\theta, \phi)=\frac{4 \pi U(\theta, \phi)}{P_{\text {in }}}
$$


If we consider a dipole planar antenna on a semi-infinite substrate with a relative dielectric constant $\left(\varepsilon_{r}\right)$, the power density ratio between the power radiating into dielectric $(\theta=\pi)$ and into the free space $(\theta=0)$ can be described by Equation 6 (Kominami et al., 1985).

$$
\frac{U(\pi, 0)}{U(0,0)}=\varepsilon_{r}{ }^{3 / 2}
$$

By considering Equations 5 and 6, the gain radiated into the dielectric can be calculated using Equation 7.

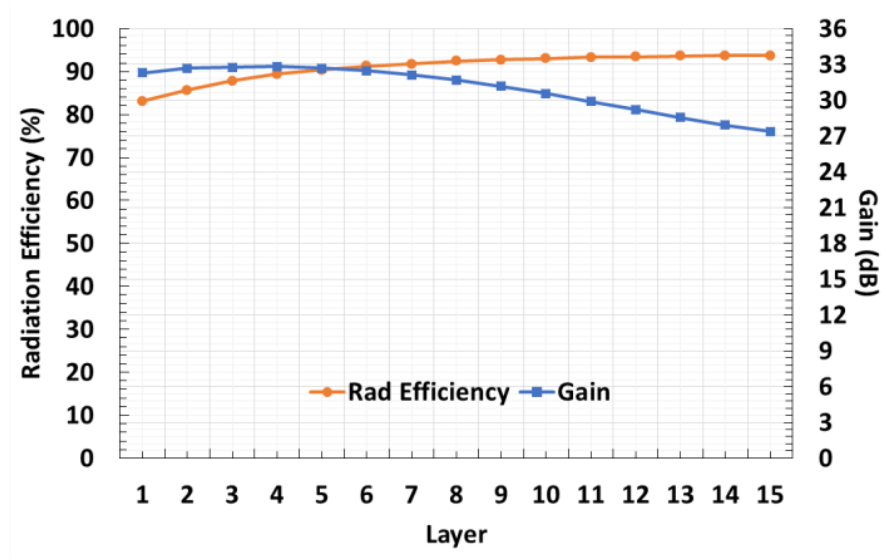

Figure 12 Simulation results of radiation efficiency and gain on the cascaded matching layer

Because it is difficult to obtain a theoretical gain of a bow-tie antenna, a dipole is considered due to its radiation pattern characteristic being similar to the bow-tie antenna. If we consider a dielectric constant of 11.9 and a dipole gain of $2.15 \mathrm{~dB}$, the gain of power radiated into the substrate is about $18.28 \mathrm{~dB}$. If an antenna is combined with a dielectric lens, this lens can increase the gain of an integrated antenna by the factor of $\varepsilon_{r}\left(n^{2}\right)$ (Rebeiz, 1992). We consider this factor to calculate the increased gain by the dielectric lens; therefore, the gain becomes $31.18 \mathrm{~dB}$.

$$
G(\pi, \phi)=\varepsilon_{r}{ }^{3 / 2} G(0, \phi)
$$

The efficiency of the transmitted power without MLs can be approximated as the transmittance of a hemispherical boundary surface. The transmittance of linear polarized wave radiation passing through a boundary can be calculated by Equations 8 and $9 . T_{p}$ is the parallel components of Fresnel's transmittance referred to a plane of incidence. $T_{s}$ is the perpendicular components referred to a plane of incidence. $\theta_{t}$ is the angle of refraction and $\theta_{t}$ is the angle of incidence. $n_{1}$ and $n_{2}$ are the refractive indices of mediums 1 and 2, respectively. For a hemispherical boundary between dielectric silicon and free space, the transmittance is $68.3 \%$ (Apriono, 2015). This level is slightly different to a planar surface, which has $70 \%$ transmittance. The efficiency of the transmitted power with MLs is ideally $100 \%$. For the same conditions in our research, efficiency by applying MLs is $93.1 \%$ (van der Vorst, 1999). The increased gain by applying MLs is $32.52 \mathrm{~dB}$, obtained by considering the increase in efficiency level.

$$
\begin{aligned}
& T_{p}=1-\left|\frac{n_{1} \cos \theta_{t}-n_{2} \cos \theta_{i}}{n_{1} \cos \theta_{t}+n_{2} \cos \theta_{i}}\right|^{2} \\
& T_{s}=1-\left|\frac{n_{1} \cos \theta_{i}-n_{2} \cos \theta_{t}}{n_{1} \cos \theta_{i}+n_{2} \cos \theta_{t}}\right|^{2}
\end{aligned}
$$


Table 3 is a summary of the gain and efficiency from the theoretical and simulation results. We compare the simulation results in the optimum conditions, which is the trade-off between gain and efficiency, as shown in Figure 12. Table 3 shows that the simulation results are comparable and consistent with the theoretical ones. The discrepancies are due to the dipole theoretical approximation rather than the bow-tie antenna.

Table 3 Theoretical and simulation result comparison of gain and radiation efficiency

\begin{tabular}{lcccc}
\hline & \multicolumn{2}{c}{ Theoretical } & \multicolumn{2}{c}{ Simulation } \\
\hline & Dielectric & Dielectric & Dielectric & Dielectric \\
& Lens & Lens + MLs & Lens & Lens + MLs \\
Realized Gain & $31.18 \mathrm{~dB}$ & $32.52 \mathrm{~dB}$ & $31.31 \mathrm{~dB}$ & $32.69 \mathrm{~dB}$ \\
Radiation Efficiency & $68.3 \%$ & $93.1 \%$ & $69.3 \%$ & $90.4 \%$ \\
\hline
\end{tabular}

\section{CONCLUSION}

A bow-tie planar antenna on a high-resistivity silicon substrate combined with a capacitive bar provided wideband bandwidth of around $368 \mathrm{GHz}$. The antenna combined with optimum substrate thickness and a hemispherical silicon lens provides gain and beam width of $31.31 \mathrm{~dB}$ and $3.1^{\circ}$, respectively. The matching layer technique of the quarter-wavelength and the five cascaded matching layers has radiation efficiency and gain of $90.4 \%$ and $32.69 \mathrm{~dB}$, respectively. The proposed design can be considered to provide a high radiation performance antenna for various $\mathrm{THz}$ applications.

\section{ACKNOWLEDGMENT}

This work was supported by PITTA 2017 Grant, contract number: 754/UN2.R3.1/HKP.05.00/2017, year 2017, Universitas Indonesia.

\section{REFERENCES}

Akyildiz, I.F., Jornet, J.M., Han, C., 2014. Terahertz Band: Next Frontier for Wireless Communications. Physical Communication. Volume 12, pp.16-32

Apriono, C., Rahardjo, E.T., Hiromoto, N., 2015. A New Method for Simulating Power Flow Density Focused by a Silicon Lens Antenna Irradiated with Linearly Polarized THz Wave. Makara Journal of Technology. Volume 19(2), pp. 59-64

Durgun, A., Balanis, C., Birtcher, C.R., Allee, D.R., 2011. Design, Simulation, Fabrication and Testing of Flexible Bow-tie Antennas. IEEE Transactions on Antennas and Propagation. Volume 59(12), pp. 4425-4435

Haraz, O.M., Abdel-Rahman, M., Alshebili, S.A., Sebak, A.R., 2014. A Novel 94-GHz Dipole Bow-tie Slot Antenna on Silicon for Imaging Applications. IEEE Asia-Pacific Conference on Applied Electromagnetics. pp. 59-62

Hesler, J.L., Liu, L., Xu, H., Duan, Y., Weikle, R., 2008. The Development of Quasi-optical THz Detectors. In: The $33^{\text {rd }}$ International Conference on Infrared, Millimeter and Terahertz Waves, pp. 1-2

Hirata, A., Kosugi, T., Takahashi, H., Takeuchi, J., Togo, H., Yaita, M., Kukutsu, N., Aihara, K., Murata, K., Sato, Y., Nagatsuma, T., Kado, Y., 2012. 120-GHz-band Wireless Link Technologies for Outdoor 10-Gbit/s Data Transmission. IEEE Transactions on Microwave Theory Techniques. Volume 60(3), pp. 881-895

International Telecommunication Union (ITU), 2009. Attenuation by Atmospheric Gases. ITUR Recommendation. pp. 676-678 
Jyothi, A., Saha, C., Ghosh, B., Kini, R., Vaisakh, C., 2016. Design of a Gain Enhanced THz Bow-tie Photoconductive Antenna. International Symposium on Antennas and Propagation. pp. 1-3

Kaur, B., Solanki, L.S., 2012. A Brief Review on Bow-tie Antenna. In: Proceeding of NCCN12, pp. 3-4

Kominami, M., Pozar, D.M., Schaubert, D.H., 1985. Dipole and Slot Elements and Arrays on Semi-infinite Substrates. IEEE Transactions on Antennas and Propagation. Volume AP33, pp. 600-607

Li, Y., Song, R., 2016. A High Gain On-chip Terahertz Antenna with High Efficiency. IEEE $9^{\text {th }}$ UK-Europe-China Workshop on Millimetre Waves and Terahertz Technologies, pp. 222224

Morita, T., Cohn, S.B., 1956. Microwave Lens Matching by Simulated Quarter Wave Transformers. IRE Transactions on Antennas and Propagation. Volume 4(1), pp. 33-39

Mourou, G., Stancampiano, C.V., Antonetti, A., Orszag, A., 1981. Picosecond Microwave Pulses Generated with a Subpicosecond Laser-driven Semiconductor Switch. Applied Physics Letters, Volume 39, p. 295

Rebeiz, G.M., 1992. Millimeter-wave and Terahertz Integrated Circuit Antennas. In: Proceedings of the IEEE. Volume 80(11), pp. 1748-1770

Ronne, C., Thrane, L., Astrand, P.O., Mikkelsen, K.V., Kediding, S.R., 1997. Investigation of the Temperature Dependence of Dielectric Relaxation in Liquid Water by $\mathrm{THz}$ Reflection Spectroscopy and Molecular Dynamics Simulation. Journal of Chemical Physics. Volume 107(14), pp. 5319-5331

Siegel, P.H., 2002. Terahertz Technology. IEEE Transactions on Microwave Theory Technology. Volume 50(3), pp. 910-928

Song, H-J., Nagatsuma, T., 2011. Present and Future of Terahertz Communications. IEEE Transaction on Terahertz Science and Technology. Volume 1(1), pp. 256-263

Tokan, F., 2014. Optimization-Based Matching Layer Design for Broadband Dielectric Lens Antennas. Applied Computational Electromagnetics Society Journal. pp. 499-507

van der Vorst, M.J.M., de Maagt, P.J.I., Herben, M.H.A.J., 1999. Effect of Internal Reflections on the Radiation Properties and Input Admittance of Integrated Lens Antennas. IEEE Transactions on Microwave Theory and Techniques. Volume 47(9), pp.1696-1704

van Houten, J.M., Herben, M.H.A.J., 1994. Analysis of a Phase Ccorrecting Fresnel-zone Plate Antenna with Dielectric/transparent Zones. Journal of Electromagnetic Waves Applications. Volume 8, pp. 847-858

Wahyudi, T., Apriono, C., Zulkifli, F.Y., Rahardjo, E.T., 2017. Broadband Planar Bow-tie Antenna on High Resistivity Silicon Substrate for Terahertz Application. In: The $15^{\text {th }}$ International Conference on Quality in Research (QiR): International Symposium on Electrical and Computer Engineering, pp. 372-376

Wang, S., Zhan, Q., 2013. Modified Bow-tie Antenna with Strong Broadband Field Enhancement for RF Photonic Applications. In: Proceedings of SPIE - The International Society for Optical Engineering, DOI. 10.1117/12.2024087

Zhang, X., Chung, C.J., Wang, S., Subbaraman, H., Pan, Z., Zhan, Q., Chen, R., 2016. Integrated Broadband Bow-tie Antenna on Transparent Silica Substrate. IEEE Antennas and Wireless Propagation Letters. Volume 15, pp. 1377-1381 